\title{
ON A NEW SHEFFER CLASS OF POLYNOMIALS RELATED TO NORMAL PRODUCT DISTRIBUTION
}

UDC 519.21

\author{
E. AZMOODEH AND D. GASBARRA
}

\begin{abstract}
In this paper, using the Stein operator $\mathfrak{R}_{\infty}$ given in [Bernoulli 23 (2017), pp. 3311-3345], associated with the normal product distribution living in the second Wiener chaos, we introduce a new class of polynomials

$$
\mathscr{P}_{\infty}:=\left\{P_{n}(x)=\mathfrak{R}_{\infty}^{n} \mathbf{1}: n \geq 1\right\} .
$$

We analyze in detail the polynomials class $\mathscr{P}_{\infty}$, and relate it to Rota's Umbral calculus by showing that it is a Sheffer family and enjoys many interesting properties. Lastly, we study the connection between the polynomial class $\mathscr{P}_{\infty}$ and the noncentral probabilistic limit theorems within the second Wiener chaos.
\end{abstract}

\section{INTRODUCTION}

The motivation of our study comes from the subsequent facts on the Gaussian distribution. Let $N \sim \mathscr{N}(0,1)$ be a standard Gaussian random variable. Consider the following well-known first order differential operator related to the so-called Ornstein-Uhlenbeck operator

$$
(L f)(x)=x f(x)-f^{\prime}(x)=-e^{x^{2} / 2} \frac{d}{d x}\left(e^{-x^{2} / 2} f\right)
$$

acting on a suitable class $\mathcal{F}$ of test functions $f$. A fundamental result in the realm of Stein method in probabilistic approximations, known as the Stein characterization of the Gaussian distribution, reads that for a given random variable $F \sim N$ if and only if $\mathrm{E}[(L f)(F)]=\mathrm{E}\left[F f(F)-f^{\prime}(F)\right]=0$ for $f \in \mathcal{F}$ (in fact, the polynomials class is enough). The second notable feature of the operator $L$ in connection with the Gaussian distribution is the following. Paul Malliavin in his book [22, p. 231], for every $n \in \mathbb{N}_{0}$, defines the so-called Hermite polynomial $H_{n}$ of order $n$ using the relation $H_{n}(x):=L^{n} \mathbf{1}(x)$. For example, the few first Hermite polynomials are given by $H_{0}(x)=1, H_{1}(x)=x$, $H_{2}(x)=x^{2}-1, H_{3}(x)=x^{3}-3 x$, and $H_{4}(x)=x^{4}-6 x+3$. One of the significant properties of the Hermite polynomials is that they constitute an orthogonal polynomials class with respect to the Gaussian measure $e^{-x^{2} / 2} / \sqrt{2 \pi} \mathrm{d} x$. The orthogonality property of the Hermite polynomials can be routinely seen as a direct consequence of the adjoint operator $L^{\star}=\frac{d}{d x}$ which is straightforward computation.

Instead of the Gaussian distribution (living in the first Wiener chaos) we consider distributions in the second Wiener chaos having a finite number of non-zero coefficients

2010 Mathematics Subject Classification. Primary 60F05, 60G50, 46L54, 60H07, 26C10.

Key words and phrases. Second Wiener chaos, normal product distribution, cumulants/moments, weak convergence, Malliavin calculus, Sheffer polynomials, umbral calculus. 
in the spectral representation, namely random variables of the form

$$
F_{\infty}=\sum_{k=1}^{d} \lambda_{k}\left(N_{k}^{2}-1\right), \quad d \geq 1
$$

where $\left(N_{k}\right)_{k \geq 1}$ is a sequence of i.i.d. $\mathscr{N}(0,1)$. Relevant examples of such random elements are centered chi-square and normal product distributions corresponding to the cases when all $\lambda_{k}$ are equal, and $d=2$ where two non-zero coefficients $\lambda_{1}=-\lambda_{2}$, respectively. The target distributions of the form (1.1) appear often in the classical framework of limit theorems of $U$-statistics; see [30, Chapter 5.5, Section 5.5.2]. Also, recently Bai and Taqqu in [8] showed that the distributions of the form (1.1) with $d=2$ can be realized as the limit in distribution of the generalized Rosenblatt process

$$
Z_{\gamma_{1}, \gamma_{2}}(t)=\int_{\mathbb{R}^{2}}^{\prime}\left(\int_{0}^{t}\left(s-x_{1}\right)_{+}^{\gamma_{1}}\left(s-x_{2}\right)_{+}^{\gamma_{2}} d s\right) B\left(d x_{1}\right) B\left(d x_{2}\right)
$$

when the exponents $\left(\gamma_{1}, \gamma_{2}\right)$ approach the boundary of the triangle

$$
\Delta:=\left\{\left(\gamma_{1}, \gamma_{2}\right) \mid-1<\gamma_{1}, \gamma_{2}<-\frac{1}{2}, \gamma_{1}+\gamma_{2}>-\frac{3}{2}\right\} .
$$

Here, $B$ stands for the Brownian measure, and the prime / indicates the off-diagonal integration. One of their interesting results reads as

$$
Z_{\gamma_{1}, \gamma_{2}}(1) \stackrel{\text { law }}{\longrightarrow} N_{1} \times N_{2} \quad \text { as }\left(\gamma_{1}, \gamma_{2}\right) \rightarrow\left(-\frac{1}{2}, \gamma\right), \quad-1<\gamma<-\frac{1}{2}
$$

Recently, the authors of [2], using two different approaches, based on Malliavin calculus and Fourier analysis, for probability distributions of the form (1.1), introduced the following so-called Stein differential operator of order $d$ (= the number of the non-zero coefficients)

$$
\mathfrak{R}_{\infty} f(x):=\sum_{l=2}^{d+1}\left(b_{l}-a_{l-1} x\right) f^{(d+2-l)}(x)-a_{d+1} x f(x),
$$

where the coefficients $\left(a_{l}\right)_{1 \leq l \leq d+1},\left(b_{l}\right)_{2 \leq l \leq d+1}$ are akin to the random element $F_{\infty}$ through the relations;

$$
a_{l}=\frac{P^{(l)}(0)}{l ! 2^{l-1}} \quad \text { and } \quad b_{l}=\sum_{r=l}^{d+1} \frac{a_{r}}{(r-l+1) !} \kappa_{r-l+2}\left(F_{\infty}\right),
$$

and $P(x)=x \prod_{i=1}^{d}\left(x-\lambda_{k}\right)$. Here $\kappa_{r}(F)$ stands for the $r$ th cumulant of the random variable $F$. In this paper, the case $d=2$ of two non-zero coefficients with the particular parametrization $\lambda_{1}=-\lambda_{2}=\frac{1}{2}$ has our interest. The operator $\mathfrak{R}_{\infty}$ given in (1.2) then reduces to

$$
\mathfrak{R}_{\infty} f(x)=\mathfrak{R} f(x):=x f(x)-f^{\prime}(x)-x f^{\prime \prime}(x) .
$$

Note that in this setup the random variable $F_{\infty}=N_{1} \times N_{2}$ (equality in distribution) is the normal product distribution. The Stein operator (1.3) associated with the normal product distribution was first introduced by Gaunt in [17. The normal product distribution also belongs to a wide class of probability distributions known as the Variance-Gamma class; consult [18] for further details and development of the Stein characterizations. Following the Gaussian framework, we define the polynomial class

$$
\mathscr{P}:=\left\{P_{n}(x):=\mathfrak{R}^{n} \mathbf{1}: n \in \mathbb{N}_{0}\right\},
$$

where operator $\mathfrak{R}$ is the same one as in (1.3). The first fifteenth polynomials $P_{n}$ are presented in Section 5 . In this short note, we study some properties of the polynomials 
class $\mathscr{P}$. We derive, among other results, that the class $\mathscr{P}$ is a Sheffer family of polynomials, hence possess a rich structure, and can be analyzed within the Gian-Carlo Rota's umbral calculus. See Subsection 3.2 for definitions. We end the note with connection of the polynomial class $\mathscr{P}$ to the non-central probabilistic limit theorems, and show that polynomial $P_{6} \in \mathscr{P}$ plays a crucial role in the limit theorems when the target distribution is the normal product random variable $N_{1} \times N_{2}$.

\section{Normal product distribution $N_{1} \times N_{2}$}

In this section, we briefly collect some properties of normal product distribution.

2.1. Modified Bessel functions of the second kind. The modified Bessel functions $I_{\nu}$ and $K_{\nu}$ with index $\nu$ of the first and the second kinds, respectively, are defined as two independent solutions of the so-called modified Bessel differential equation

$$
\left[\Re_{M B}^{\nu} f\right](x):=x^{2} f^{\prime \prime}(x)+x f^{\prime}(x)-\left(x^{2}+\nu^{2}\right) f(x)=0
$$

with the convention $\mathfrak{R}_{M B}^{0}=\mathfrak{R}_{M B}$. We collect the following results on the modified Bessel function of the second kind and the normal product distribution.

(i) It is well known that (see, e.g., [32]) the density function $p_{\infty}$ of the normal product random variable is given by

$$
p_{\infty}(x)=\frac{1}{\pi} K_{0}(|x|), \quad x \in \mathbb{R},
$$

where $K_{0}$ is the modified Bessel function of the second kind with the index $\nu=0$.

(ii) The modified Bessel function of the second kind $K_{0}$ possesses several useful representations. Among those, here we state

$$
K_{0}(|x|)=\frac{1}{2} G_{0,2}^{2,0}\left(\frac{x^{2}}{4} \mid 0,0\right),
$$

where $G$ here is the so-called Meijer G-function that shares many interesting properties; see, e.g., 1 .

(iii) $\frac{d}{d x} K_{0}(x)=-K_{1}(x), K_{0}(x) \sim-\log (x)$ as $x \downarrow 0$, and

$$
K_{0}(x) \sim \sqrt{\frac{\pi}{2 x}} e^{-x} \text { as } x \rightarrow \infty .
$$

(iv) The relation $-x[\mathfrak{R} f](x)=x^{2} f^{\prime \prime}(x)+x f(x)-x^{2} f(x)=\left[\mathfrak{R}_{M B} f\right](x)$ holds, where $\mathfrak{R}$ as in (1.3) is the Stein operator associated with the normal product distribution.

(v) The characteristic function of the normal product distribution is

$$
\varphi_{\infty}(t)=\left(1+t^{2}\right)^{-1}
$$

Hence, the normal product distribution is the unique random variable in the second Wiener chaos having only two non-zero coefficients equal with

$$
\lambda_{1}=-\lambda_{2}=\frac{1}{2}
$$

(vi) For $n \in \mathbb{N}, \mu_{2 n}\left(N_{1} \times N_{2}\right):=\mathrm{E}\left[\left(N_{1} \times N_{2}\right)^{2 n}\right]=((2 n-1) ! !)^{2}, \kappa_{2 n}\left(N_{1} \times N_{2}\right)=$ $(2 n-1)$ ! and $\mu_{2 n-1}\left(N_{1} \times N_{2}\right)=\kappa_{2 n-1}\left(N_{1} \times N_{2}\right)=0$. 
2.2. The adjoint operator $\mathfrak{R}^{\star}$. We recall the following well-known finite dimensional Gaussian integration by parts formulae. For a detailed discussion, the reader is referred to the lecture note written by Vlad Bally [9].

Lemma 2.1. Let $N_{1}, \ldots, N_{T}$ be i.i.d. $\mathscr{N}(0,1)$. For smooth random variables

$$
F\left(N_{1}, \ldots, N_{T}\right) \quad \text { and } \quad\left(u_{t}\left(N_{1}, \ldots, N_{T}\right)\right)_{t=1, \ldots T}
$$

we have the finite dimensional Gaussian integration by parts formula

$$
\mathrm{E}(\langle D F, u\rangle)=\mathrm{E}(F \delta(u))
$$

where $D$ is the gradient operator and

$$
\delta(u)=\sum_{t=1}^{T} u_{t} N_{t}-\sum_{t=1}^{T} D_{t} u_{t}
$$

denotes the Skorokhod integral in the finite dimension.

Proposition 2.1. Let $F_{\infty}=N_{1} \times N_{2}$, and let $\mu_{\infty}=p_{\infty} d x$ be the associated probability measure on the real line. Consider the second order differential operator

$$
(\Re f)(x)=x f(x)-f^{\prime}(x)-x f^{\prime \prime}(x) .
$$

Then the adjoint operator $\mathfrak{R}^{\star}$ in the space $L^{2}\left(\mathbb{R}, \mu_{\infty}\right)$ is

$$
\left(\mathfrak{R}^{*} g\right)(x)=(\mathfrak{R} g)(x)+\theta(x) g^{\prime}(x)-x g(x),
$$

where the special function $\theta$ is given by the conditional expectation

$$
\theta(x)=\mathrm{E}\left(N_{1}^{2}+N_{2}^{2} \mid N_{1} N_{2}=x\right)=2|x| \frac{K_{1}(|x|)}{K_{0}(|x|)} .
$$

Proof. In order to compute the adjoint of $\Re^{\star}$, we use Lemma 2.1 with $u=\left(\begin{array}{l}u_{1} \\ u_{2}\end{array}\right)=\frac{1}{2}\left(\begin{array}{l}N_{1} \\ N_{2}\end{array}\right)$ and $v=\frac{1}{2}\left(\begin{array}{l}N_{2} \\ N_{1}\end{array}\right)$. We write

$$
\begin{aligned}
\mathrm{E}\left(f^{\prime \prime}\right. & \left.\left(N_{1} N_{2}\right) g\left(N_{1} N_{2}\right) N_{1} N_{2}\right)=\mathrm{E}\left(\left\langle D f^{\prime}\left(N_{1} N_{2}\right), g\left(N_{1} N_{2}\right) u\right\rangle\right) \\
& =\mathrm{E}\left(f^{\prime}\left(N_{1} N_{2}\right) \delta\left(g\left(N_{1} N_{2}\right) u\right)\right) \\
& =\mathrm{E}\left(f^{\prime}\left(N_{1} N_{2}\right)\left(g\left(N_{1} N_{2}\right) \frac{\left(N_{1}^{2}+N_{2}^{2}\right)}{2}-g^{\prime}\left(N_{1} N_{2}\right) N_{1} N_{2}-g\left(N_{1} N_{2}\right)\right)\right),
\end{aligned}
$$

where

$$
\begin{aligned}
& \mathrm{E}\left(f^{\prime}\left(N_{1} N_{2}\right) g^{\prime}\left(N_{1} N_{2}\right) N_{1} N_{2}\right) \\
& \quad=\mathrm{E}\left(f\left(N_{1} N_{2}\right)\left(g^{\prime}\left(N_{1} N_{2}\right) \frac{\left(N_{1}^{2}+N_{2}^{2}\right)}{2}-g^{\prime \prime}\left(N_{1} N_{2}\right) N_{1} N_{2}-g^{\prime}\left(N_{1} N_{2}\right)\right)\right)
\end{aligned}
$$

and

$$
\begin{gathered}
\mathrm{E}\left(f^{\prime}\left(N_{1} N_{2}\right)\left(g\left(N_{1} N_{2}\right) \frac{\left(N_{1}^{2}+N_{2}^{2}\right)}{2}\right)\right)=\mathrm{E}\left(\left\langle D f\left(N_{1} N_{2}\right), g\left(N_{1} N_{2}\right) v\right\rangle\right) \\
\quad=\mathrm{E}\left(f\left(N_{1} N_{2}\right) \delta\left(g\left(N_{1} N_{2}\right) v\right)\right) \\
\quad=\mathrm{E}\left(f\left(N_{1} N_{2}\right)\left(g\left(N_{1} N_{2}\right) N_{1} N_{2}-g^{\prime}\left(N_{1} N_{2}\right) \frac{\left(N_{1}^{2}+N_{2}^{2}\right)}{2}\right)\right) .
\end{gathered}
$$


Therefore we have

$$
\begin{aligned}
\mathrm{E}(\Re)( & \left.\left.N_{1} N_{2}\right) g\left(N_{1} N_{2}\right)\right) \\
= & \mathrm{E}\left(f\left(N_{1} N_{2}\right) g\left(N_{1} N_{2}\right) N_{1} N_{2}\right)-\mathrm{E}\left(f^{\prime}\left(N_{1} N_{2}\right) g\left(N_{1} N_{2}\right)\right) \\
& -\mathrm{E}\left(f^{\prime \prime}\left(N_{1} N_{2}\right) g\left(N_{1} N_{2}\right) N_{1} N_{2}\right) \\
= & \mathrm{E}\left(f\left(N_{1} N_{2}\right) g\left(N_{1} N_{2}\right) N_{1} N_{2}\right)-\mathrm{E}\left(f^{\prime}\left(N_{1} N_{2}\right) g\left(N_{1} N_{2}\right)\right) \\
& +\mathrm{E}\left(f\left(N_{1} N_{2}\right)\left(g^{\prime}\left(N_{1} N_{2}\right) \frac{\left(N_{1}^{2}+N_{2}^{2}\right)}{2}-g^{\prime \prime}\left(N_{1} N_{2}\right) N_{1} N_{2}-g^{\prime}\left(N_{1} N_{2}\right)\right)\right) \\
& +\mathrm{E}\left(f^{\prime}\left(N_{1} N_{2}\right) g\left(N_{1} N_{2}\right)\right) \\
& -\mathrm{E}\left(f\left(N_{1} N_{2}\right)\left(g\left(N_{1} N_{2}\right) N_{1} N_{2}-g^{\prime}\left(N_{1} N_{2}\right) \frac{\left(N_{1}^{2}+N_{2}^{2}\right)}{2}\right)\right) \\
= & \mathrm{E}\left(f\left(N_{1} N_{2}\right)\left(g^{\prime}\left(N_{1} N_{2}\right)\left(N_{1}^{2}+N_{2}^{2}\right)-g^{\prime \prime}\left(N_{1} N_{2}\right) N_{1} N_{2}-g^{\prime}\left(N_{1} N_{2}\right)\right)\right) \\
= & \mathrm{E}\left(f\left(N_{1} N_{2}\right)\left(g^{\prime}\left(N_{1} N_{2}\right) \mathrm{E}\left(N_{1}^{2}+N_{2}^{2} \mid N_{1} N_{2}\right)-g^{\prime \prime}\left(N_{1} N_{2}\right) N_{1} N_{2}-g^{\prime}\left(N_{1} N_{2}\right)\right)\right) .
\end{aligned}
$$

This implies that

$$
\mathfrak{R}^{*} g(x)=\eta(x) g^{\prime}(x)-g^{\prime \prime}(x) x,
$$

where the conditional expectation

$$
\eta(x)=\mathrm{E}\left(N_{1}^{2}+N_{2}^{2} \mid N_{1} N_{2}=x\right)-1
$$

is a special function. In order to compute explicitly the special function $\eta$, we make use of Lemma 6.1 to write

$$
\eta(x)=\mathrm{E}\left(N_{1}^{2}+N_{2}^{2} \mid N_{1} N_{2}=x\right)-1=\frac{\mathrm{E}\left(\delta_{x}\left(N_{1} N_{2}\right)\left(N_{1}^{2}+N_{2}^{2}\right)\right)}{p_{\infty}(x)}-1,
$$

where

$$
\begin{aligned}
\mathrm{E}\left(\left(N_{1}^{2}+N_{2}^{2}\right) \delta_{x}\left(N_{1} N_{2}\right)\right) & =\frac{1}{2 \pi} \int_{\mathbb{R}}\left(\int_{\mathbb{R}}\left(z^{2}+y^{2}\right) \delta_{x}(z y) e^{-z^{2} / 2} d z\right) e^{-y^{2} / 2} d y \\
& =\frac{1}{2 \pi} \int_{\mathbb{R}}\left(\int_{\mathbb{R}}\left(\frac{u^{2}}{y^{2}}+y^{2}\right) \delta_{x}(u) e^{-u^{2} /\left(2 y^{2}\right)} y^{-1} d u\right) e^{-y^{2} / 2} d y \\
& =\frac{1}{2 \pi} \int_{\mathbb{R}}\left(\frac{x^{2}}{y^{2}}+y^{2}\right) e^{-x^{2} /\left(2 y^{2}\right)}|y|^{-1} e^{-y^{2} / 2} d y \\
& =\frac{1}{2 \pi} \int_{0}^{\infty}\left(\frac{x^{2}}{v}+v\right) e^{-x^{2} /(2 v)-v / 2} v^{-1} d v \\
& =\frac{2|x| K_{1}(|x|)}{\pi}
\end{aligned}
$$

by changing variables first with $z=u / y$ and then with $v=y^{2}$. Therefore

$$
\eta(x)=\frac{2|x| K_{1}(|x|)-K_{0}(|x|)}{K_{0}(|x|)},
$$

and the result follows at once taking into account the definition of operator $\mathfrak{R}$.

3. The POLYNOMial Class $\mathscr{P}$

3.1. Some basic properties of polynomials $P_{n}$. Recall that

$$
\mathscr{P}:=\left\{P_{n}(x):=\mathfrak{R}^{n} \mathbf{1}: n \geq 0\right\},
$$


where the Stein operator $\mathfrak{R}$ associated to the normal product random variable $N_{1} \times N_{2}$ is given by the second order differential operator

$$
\mathfrak{R} f(x)=x f(x)-f^{\prime}(x)-x f^{\prime \prime}(x) .
$$

We start with the following observation on the coefficients of polynomials $P_{n}$.

Proposition 3.1. For every $n \geq 1$, the polynomial $P_{n} \in \mathscr{P}$ is of degree $n$. Also, assume that

$$
P_{n}(x):=\sum_{k=0}^{n} \mathfrak{a}(n, k) x^{k}, \quad n \geq 1 .
$$

Then, for $n \in \mathbb{N}$, the following properties hold:

(i) $\mathfrak{a}(n, n)=1$, i.e., all polynomials $P_{n}$ are monic.

(ii) $\mathfrak{a}(n, n-(2 k-1))=0$ for all $1 \leq k \leq\left[\frac{n+1}{2}\right]$. In particular, $\mathfrak{a}(n, n-1)=0$.

(iii) The doubly indices sequence $\mathfrak{a}(n, k)$ satisfies in the recursive relation

$$
\mathfrak{a}(n, k)=\mathfrak{a}(n-1, k-1)-(k+1)^{2} \mathfrak{a}(n-1, k+1)
$$

with two terminal conditions $\mathfrak{a}(n, n)=1, \mathfrak{a}(n, n-1)=0$. Moreover, the solution of recursive formula (3.2) for every $0<k \leq \frac{n}{2}$ ( or $\frac{n-1}{2}$ ) depending on whether $n$ is even or $n$ is odd, is given by

$$
\mathfrak{a}(n, n-2 k)=(-1)^{k} \sum_{i_{1}=1}^{n-2 k+1} i_{1}^{2} \sum_{i_{2}=1}^{i_{1}+1} i_{2}^{2} \sum_{i_{3}=1}^{i_{2}+1} i_{3}^{2} \cdots \sum_{i_{k}=1}^{i_{k-1}+1} i_{k}^{2} .
$$

(iv) For every $n \in \mathbb{N}$,

$$
\mathfrak{a}(n, 0)= \begin{cases}0 & \text { if } n \text { is odd, } \\ (-1)^{n / 2} \sum_{i_{1}=1}^{1} i_{1}^{2} \sum_{i_{2}=1}^{i_{1}+1} i_{2}^{2} \sum_{i_{3}=1}^{i_{2}+1} i_{3}^{2} \cdots \sum_{i_{n / 2}=1}^{i_{n / 2-1}+1} i_{n / 2}^{2} & \text { if } n \text { is even } .\end{cases}
$$

(v) For $n$ even,

$$
\mathfrak{a}(n, 0)=4 \sum_{k=1}^{n / 2}(-1)^{k-1} \mathfrak{a}(n-2 k, 2) .
$$

Proof. (i) It is straightforward (for example, by an induction argument on $n$ ) to see that for every $n \in \mathbb{N}$, we have $\operatorname{deg}\left(P_{n}\right)=n$, and moreover $\mathfrak{a}(n, n)=1$. (ii) We again proceed with an induction argument on $(n, k)$ for $0 \leq k \leq n$, and $n \in \mathbb{N}$. Obviously, the claim holds for starting values $n=1,2$. Assume that it holds for some $n \in \mathbb{N}$ that $\mathfrak{a}(n, n-(2 k-1))=0$ for all $1 \leq k \leq\left[\frac{n+1}{2}\right]$. We want to show it also holds for $n+1$. Using the very definition of polynomial

$$
P_{n+1}(x)=\mathfrak{R} P_{n}(x)=x P_{n}(x)-P_{n}^{\prime}(x)-x P_{n}^{\prime \prime}(x),
$$

and doing some simple computation we infer that

$$
\mathfrak{a}(n+1, k)=\mathfrak{a}(n, k-1)-(k+1)^{2} \mathfrak{a}(n, k+1)
$$

for $0 \leq k \leq n+1$ with the convention that $\mathfrak{a}(n, k)=0$ for every value $0>k>n$. Now the claim for $n+1$ easily follows from induction hypothesis. (iii) Using recursive 
relation (3.2) we can infer that

$$
\begin{aligned}
\mathfrak{a}(n, n-2 k)= & \mathfrak{a}(n-1,(n-1)-2 k)-(n-2 k+1)^{2} \mathfrak{a}(n-1,(n-1)-2(k-1)) \\
= & \left\{(-1)^{k} \sum_{i_{1}=1}^{n-2 k} i_{1}^{2} \sum_{i_{2}=1}^{i_{1}+1} i_{2}^{2} \sum_{i_{3}=1}^{i_{2}+1} i_{3}^{2} \ldots \sum_{i_{k}=1}^{i_{k-1}+1} i_{k}^{2}\right\} \\
& -\left\{(-1)^{k-1}(n-2 k+1)^{2} \sum_{i_{1}=1}^{n-2 k+2} i_{1}^{2} \sum_{i_{2}=1}^{i_{1}+1} i_{2}^{2} \sum_{i_{3}=1}^{i_{2}+1} i_{3}^{2} \ldots \sum_{i_{k-1}=1}^{i_{k-2}+1} i_{k-1}^{2}\right\} \\
= & (-1)^{k}\left\{\left\{\sum_{i_{1}=1}^{n-2 k} i_{1}^{2} \sum_{i_{2}=1}^{i_{1}+1} i_{2}^{2} \sum_{i_{3}=1}^{i_{2}+1} i_{3}^{2} \ldots \sum_{i_{k}=1}^{i_{k-1}+1} i_{k}^{2}\right\}\right. \\
& \left.+\left\{(n-2 k+1)^{2} \sum_{i_{1}=1}^{n-2 k+2} i_{1}^{2} \sum_{i_{2}=1}^{i_{1}+1} i_{2}^{2} \sum_{i_{3}=1}^{i_{2}+1} i_{3}^{2} \ldots \sum_{i_{k-1}=1}^{i_{k-2}+1} i_{k-1}^{2}\right\}\right\} \\
= & (-1)^{k} \sum_{i_{1}=1}^{n-2 k+1} i_{1}^{2} \sum_{i_{2}=1}^{i_{1}+1} i_{2}^{2} \sum_{i_{3}=1}^{i_{2}+1} i_{3}^{2} \ldots \sum_{i_{k}=1}^{i_{k-1}+1} i_{k}^{2} .
\end{aligned}
$$

(iv) This part is a direct consequence of item (iii), and finally (v) can be obtained directly from the recursive relation (3.2).

Lemma 3.1. Let $m, n \in \mathbb{N}$. For polynomials $P_{n} \in \mathscr{P}$, the following properties hold:

(i) $\left(\mathfrak{R} P_{n-1}^{\prime}\right)(x)=P_{n-1}^{\prime \prime}(x)-P_{n-1}(x)+P_{n}^{\prime}(x)$.

(ii) $\mathfrak{R}\left(P_{n} P_{m}\right)=P_{n} \mathfrak{R} P_{m}+P_{m} \mathfrak{R} P_{n}-\left(2 x P_{n}^{\prime} P_{m}^{\prime}+x P_{n} P_{m}\right)$. In particular,

$$
\mathfrak{R}\left(x P_{n}\right)=x P_{n+1}-P_{n}-2 x P_{n}^{\prime} .
$$

(iii) $\mathrm{E}\left[P_{n}\left(F_{\infty}\right)\right]=0$.

(iv) For every $n \in \mathbb{N}$, it holds that

$$
\mathrm{E}\left[F_{\infty} P_{n+1}\left(F_{\infty}\right)\right]=2 \mathrm{E}\left[F_{\infty} P_{n}^{\prime}\left(F_{\infty}\right)\right]
$$

In particular, when $n$ is odd both sides vanish.

(v) Let $n$ be even, and $m$ odd, or vice versa. Then $\mathrm{E}\left[P_{n}\left(F_{\infty}\right) P_{m}\left(F_{\infty}\right)\right]=0$. In particular,

$$
\mathrm{E}\left[P_{n}\left(F_{\infty}\right)\left(\Re P_{n}\right)\left(F_{\infty}\right)\right]=\mathrm{E}\left[P_{n}\left(F_{\infty}\right) P_{n+1}\left(F_{\infty}\right)\right]=0
$$

This item provides some sort of "weak orthogonality".

Proof. By the very definition of polynomials in the class $\mathscr{P}$ using raising operator $\mathfrak{R}$ it yields that $P_{n}=x P_{n-1}-P_{n-1}^{\prime}-x P_{n-1}^{\prime \prime}$ for every $n \geq 1$. Then items (i), (ii) can be obtained by doing some straightforward computations, using the definition of $\mathfrak{R}$ operator, and $P_{1}(x)=x$. (iii) It holds that $\mathrm{E}\left[P_{n}\left(F_{\infty}\right)\right]=\mathrm{E}\left[\mathfrak{R}\left(P_{n-1}\right)\left(F_{\infty}\right)\right]$, and the latter expectation vanishes since the raising operator $\mathfrak{R}$ serves as an associated Stein operator for the normal product distribution. However, to be self-contained, here we present a simple proof based on Lemma 2.1. Let $u=\left(u_{1} u_{2}\right)^{t}=\frac{1}{2}\left(N_{1} N_{2}\right)^{t}$, so $\delta(u)=\left(N_{1}^{2}+N_{2}^{2}\right) / 2-1$. By using the Gaussian integration by parts formula on $L^{2}\left(\mathbb{R}^{2}, \gamma \otimes \gamma\right)$, where $\gamma$ is the 
standard Gaussian measure, we write

$$
\begin{aligned}
\mathrm{E}\left(f^{\prime \prime}\left(N_{1} N_{2}\right) N_{1} N_{2}\right) & =\mathrm{E}\left(\left\langle D f^{\prime}\left(N_{1} N_{2}\right), u\right\rangle\right)=\mathrm{E}\left(f^{\prime}\left(N_{1} N_{2}\right) \delta(u)\right) \\
& =\mathrm{E}\left(f^{\prime}\left(N_{1} N_{2}\right)\left(\frac{N_{1}^{2}+N_{2}^{2}}{2}-1\right)\right) \\
& =-\mathrm{E}\left(f^{\prime}\left(N_{1} N_{2}\right)\right)+\mathrm{E}\left(\left\langle D f\left(N_{1} N_{2}\right), v\right\rangle\right) \\
& =-\mathrm{E}\left(f^{\prime}\left(N_{1} N_{2}\right)\right)+\mathrm{E}\left(f\left(N_{1} N_{2}\right) \delta(v)\right) \\
& =-\mathrm{E}\left(f^{\prime}\left(N_{1} N_{2}\right)\right)+\mathrm{E}\left(f\left(N_{1} N_{2}\right) N_{1} N_{2}\right), \\
& v=\left(\begin{array}{c}
v_{1} \\
v_{2}
\end{array}\right)=\frac{1}{2}\left(\begin{array}{l}
N_{2} \\
N_{1}
\end{array}\right),
\end{aligned}
$$

since $\delta(v)=N_{1} N_{2}$ for every function $f$ as soon as the involved expectations exist, in particular the polynomial functions. (iv) It is a direct consequence of items (ii), (iii), and the fact that $P_{n}(x)=\Re P_{n-1}(x)$. (v) Note that

$$
\mathrm{E}\left[P_{n}\left(F_{\infty}\right)\left(\Re P_{m}\right)\left(F_{\infty}\right)\right]=\mathrm{E}\left[P_{n}\left(F_{\infty}\right) P_{m+1}\left(F_{\infty}\right)\right],
$$

and the latter expectation also vanishes relying on item (ii) of Lemma 3.1, and the fact that the random variable $F_{\infty}$ is a symmetric distribution yields that all the odd moments $F_{\infty}$ vanish.

We continue with the non-orthogonality result of the polynomials family $\mathscr{P}$.

Proposition 3.2. The family $\mathscr{P}$ is not a class of orthogonal polynomials.

Proof. Using the Favard Theorem (see [14]), if the family $\mathscr{P}$ would be a class of orthogonal polynomials, then there exist numerical constants $c_{n}, d_{n}$ so that for polynomial $P_{n} \in \mathscr{P}$, we have

$$
P_{n+1}(x)=\left(x-c_{n}\right) P_{n}(x)-d_{n} P_{n-1}(x) .
$$

On the other hand, by the very definition of operator $\mathfrak{R}$, we also have the following relation:

$$
P_{n+1}(x)=x P_{n}(x)-P_{n}^{\prime}(x)-x P_{n}^{\prime \prime}(x) .
$$

Hence, $c_{n} P_{n}(x)=P_{n}^{\prime}(x)+x P_{n}^{\prime \prime}(x)-d_{n} P_{n-1}(x)$. Now, taking into account that $\operatorname{deg}\left(P_{n}\right)=$ $n$ for all $n \in \mathbb{N}$, when $c_{n} \neq 0$, a degree argument leads to a contradiction. If $c_{n_{0}}=0$ for some $n_{0} \in \mathbb{N}$, then we have necessarily $d_{n_{0}}=n_{0}^{2}$; see also Remark 3.2 This is because of the fact that all polynomials $P_{n}$ are monic. Now, assume that

$$
P_{n-1}(x)=\sum_{k=0}^{n-1} \mathfrak{a}(n-1, k) x^{k}, \quad \mathfrak{a}(n-1, n-1)=1 .
$$

Then, using the definition of polynomial $P_{n}$ through the operator $\mathfrak{R}$, one can obtain that

$$
\begin{aligned}
P_{n}(x)= & x^{n}+\mathfrak{a}(n-1, n-2) x^{n-1} \\
& +\sum_{k=1}^{n-2}\left(\mathfrak{a}(n-1, k-1)-(k+1)^{2} \mathfrak{a}(n-1, k+1)\right) x^{k}-\mathfrak{a}(n-1,1) .
\end{aligned}
$$

On the other hand, relation $n_{0}^{2} P_{n_{0}-1}(x)=P_{n_{0}}^{\prime}(x)+x P_{n_{0}}^{\prime \prime}(x)$ implies that

$$
P_{n_{0}+1}(x)=x P_{n_{0}}(x)-n_{0}^{2} P_{n_{0}-1} \text {. }
$$

Hence,

$$
\mathfrak{a}\left(n_{0}+1,0\right)=4 \mathfrak{a}\left(n_{0}-1,2\right)-\mathfrak{a}\left(n_{0}-1,0\right)=n_{0}^{2} \mathfrak{a}\left(n_{0}-1,0\right),
$$

i.e., $4 \mathfrak{a}\left(n_{0}-1,2\right)=\left(1+n_{0}^{2}\right) \mathfrak{a}\left(n_{0}-1,0\right)$, which also leads to a contradiction, because one side is positive and the other side negative. 
Remark 3.1. It is worth noting that it is easy to see the class $\mathscr{P}$ is not orthogonal with respect to probability measure induced by random variable $F_{\infty}$. For example, we have $\mathrm{E}\left[P_{2}\left(F_{\infty}\right) \times P_{4}\left(F_{\infty}\right)\right]=94 \neq 0$. See also item $(\mathrm{v})$ of Lemma 3.1

We close this section with a neat application of the adjoint operator $\mathfrak{R}^{\star}$. We present the following average version of the well-known Turan's inequality in the framework of orthogonal polynomials.

Proposition 3.3. For every $n \in \mathbb{N}$, the following inequality holds:

$$
\mathrm{E}\left[P_{n}^{2}\left(F_{\infty}\right)\right] \geq \mathrm{E}\left[P_{n-1}\left(F_{\infty}\right) P_{n+1}\left(F_{\infty}\right)\right] .
$$

Proof. According to Proposition 2.1, we can write

$$
\begin{aligned}
\mathrm{E}\left[P_{n}^{2}\left(F_{\infty}\right)\right]= & \mathrm{E}\left[P_{n}\left(F_{\infty}\right) \mathfrak{L} P_{n-1}\left(F_{\infty}\right)\right]=\mathrm{E}\left[P_{n-1}\left(F_{\infty}\right) \mathfrak{L}^{\star} P_{n}\left(F_{\infty}\right)\right] \\
= & \mathrm{E}\left[P_{n-1}\left(F_{\infty}\right) \mathfrak{L} P_{n}\left(F_{\infty}\right)\right]+\mathrm{E}\left[P_{n-1}\left(F_{\infty}\right) \theta\left(F_{\infty}\right) P_{n}^{\prime}\left(F_{\infty}\right)\right] \\
& -\mathrm{E}\left[F_{\infty} P_{n-1}\left(F_{\infty}\right) P_{n}\left(F_{\infty}\right)\right] \\
= & \mathrm{E}\left[P_{n-1}\left(F_{\infty}\right) P_{n+1}\left(F_{\infty}\right)\right]+\mathrm{E}\left[P_{n-1}\left(F_{\infty}\right) \theta\left(F_{\infty}\right) P_{n}^{\prime}\left(F_{\infty}\right)\right] \\
& -\mathrm{E}\left[F_{\infty} P_{n-1}\left(F_{\infty}\right) P_{n}\left(F_{\infty}\right)\right],
\end{aligned}
$$

where the special function

Hence, we are left to show that

$$
\theta(x)=2|x| \frac{K_{1}(|x|)}{K_{0}(|x|)}
$$

$$
\mathrm{E}\left[P_{n-1}\left(F_{\infty}\right) \theta\left(F_{\infty}\right) P_{n}^{\prime}\left(F_{\infty}\right)\right] \geq \mathrm{E}\left[F_{\infty} P_{n-1}\left(F_{\infty}\right) P_{n}\left(F_{\infty}\right)\right] .
$$

The latter is equivalent to showing that

$$
2 \int_{0}^{\infty} x P_{n-1}(x) P_{n}^{\prime}(x) K_{1}(x) d x \geq \int_{0}^{\infty} x P_{n-1}(x) P_{n}(x) K_{0}(x) d x .
$$

We also need the following integral formula taken from Gradshetyn and Ryzhik [21, p. 676]:

$$
\int_{0}^{\infty} x^{\mu} K_{\nu}(x) d x=2^{\mu-1} \Gamma\left(\frac{1+\mu+\nu}{2}\right) \Gamma\left(\frac{1+\mu-\nu}{2}\right), \quad \Re(\mu+1 \pm \nu)>0 .
$$

Now assume that $P_{n}(x)=\sum_{k=0}^{n} \mathfrak{a}(n, k) x^{k}$. Then using the integral formula (3.10) together with some straightforward computation, inequality (3.9) is equivalent to showing that

$$
\begin{aligned}
2 \sum_{k=1}^{2 n-1} & \sum_{l=0}^{k} l \mathfrak{a}(n, l) \mathfrak{a}(n-1, k-l) 2^{k-1} \Gamma\left(\frac{2+k}{2}\right) \Gamma\left(\frac{k}{2}\right) \\
& =2 \sum_{k=1}^{2 n-1} \sum_{l=0}^{k} l \mathfrak{a}(n, l) \mathfrak{a}(n-1, k-l) 2^{k-1} k\left[\frac{(k-2) ! ! \sqrt{\pi}}{2^{k / 2}}\right]^{2} \\
& \geq \sum_{k=1}^{2 n} \sum_{l=0}^{k} \mathfrak{a}(n, l) \mathfrak{a}(n-1, k-l-1) 2^{k-1} \Gamma\left(\frac{1+k}{2}\right) \Gamma\left(\frac{1+k}{2}\right) \\
& =\sum_{k=1}^{2 n} \sum_{l=0}^{k} \mathfrak{a}(n, l) \mathfrak{a}(n-1, k-l-1) 2^{k-1}\left[\frac{(k-1) ! ! \sqrt{\pi}}{2^{k / 2}}\right]^{2} .
\end{aligned}
$$

We set

$$
A(n, k)=(k ! !)^{2} \sum_{l=0}^{k} \frac{l}{k} \mathfrak{a}(n, l) \mathfrak{a}(n-1, k-l), \quad B(n, k)=\frac{(k ! !)^{2}}{2} \sum_{l=0}^{k} \mathfrak{a}(n, l) \mathfrak{a}(n-1, k-l) .
$$


Hence, we are left to show that

$$
\sum_{1 \leq k \leq 2 n-1, k \text { odd }} A(n, k) \geq \sum_{1 \leq k \leq 2 n-1, k \text { odd }} B(n, k) .
$$

The last inequality itself can be shown by using induction on $n$, together with some straightforward but tedious computations, the recursive relation (3.2), and the shape of coefficients $\mathfrak{a}(n, k)$ given by relation (3.3).

3.2. Generating function and the Sheffer sequences. In this section, we provide some fundamental elements of theory of Sheffer class polynomials. For a complete overview, the reader may consult the monograph [27. Sequences of polynomials play a fundamental role in mathematics. One of the most famous classes of polynomial sequences is the class of Sheffer sequences, which contains many important sequences such as those formed by Bernoulli polynomials, Euler polynomials, Abel polynomials, Hermite polynomials, Laguerre polynomials, etc., and contains the classes of associated sequences and Appell sequences as two subclasses. Roman et al. in [27, 29] studied the Sheffer sequences systematically by the theory of modern umbral calculus.

Let $\mathbb{K}$ be a field of characteristic zero. Let $\mathcal{F}$ be the set of all formal power series in the variable $t$ over $\mathbb{K}$. Thus an element of $\mathcal{F}$ has the form

$$
f(t)=\sum_{k=0}^{\infty} a_{k} t^{k}
$$

where $a_{k} \in \mathbb{K}$ for all $k \in \mathbb{N}:=\{0,1,2, \ldots\}$. The order $\mathrm{O}(f(t))$ of a power series $f(t)$ is the smallest integer $k$ for which the coefficient of $t^{k}$ does not vanish. The series $f(t)$ has a multiplicative inverse, denoted by $f(t)^{-1}$ or $\frac{1}{f(t)}$, if and only if $\mathrm{O}(f(t))=0$. Then $f(t)$ is called an invertible series. The series $f(t)$ has a compositional inverse, denoted by $\bar{f}(t)$ and satisfying $f(\bar{f}(t))=\bar{f}(f(t))=t$, if and only if $\mathrm{O}(f(t))=1$. Then $f(t)$ is called a delta series. Let $f(t)$ be a delta series and let $g(t)$ be an invertible series of the following forms:

$$
f(t)=\sum_{n=0}^{\infty} f_{n} \frac{t^{n}}{n !}, \quad f_{0}=0, f_{1} \neq 0
$$

and

$$
g(t)=\sum_{n=0}^{\infty} g_{n} \frac{t^{n}}{n !}, \quad g_{0} \neq 0 .
$$

Moreover, for any formal power series $f$ of the form (3.11), the action of $f$ on the monomials is defined by $\left\langle f \mid x^{n}\right\rangle=a_{n}$ for all $n \geq 0$. By linearity, the definition can be easily extended to a general polynomial $s(x)$.

Theorem 3.1 (Sheffer sequence [27, Theorem 2.3.1]). Let $f(t)$ be a delta series and let $g(t)$ be an invertible series. Then there exists a unique sequence $s_{n}(x)$ of polynomials satisfying the orthogonality conditions

$$
\left\langle g(t) f(t)^{k} \mid s_{n}(x)\right\rangle=c_{n} \delta_{n, k}
$$

for all $n, k \geq 0$, where $\delta_{n, k}$ is the Kronecker delta, and $\langle L \mid p(x)\rangle$ denotes the action of a linear functional $L$ on a polynomial $p(x)$. In this case, we say that the sequence $s_{n}(x)$ in (3.12) is the Sheffer sequence for the pair $(g(t), f(t))$, or that $s_{n}(x)$ is Sheffer for $(g(t), f(t))$. In particular, the Sheffer sequence for $(1, f(t))$ is called the associated 
sequence for $f(t)$ defined by the generating function of the form

$$
e^{x \bar{f}(t)}=\sum_{n=0}^{\infty} \tilde{s}_{n}(x) \frac{t^{n}}{n !}
$$

and the Sheffer sequence for $(g(t), t)$ is called the Appell sequence for $g(t)$ defined by the generating function of the form

$$
\frac{1}{g(t)} e^{x t}=\sum_{n=0}^{\infty} A_{n}(x) \frac{t^{n}}{n !} .
$$

Theorem 3.2 ([27, Theorem 2.3.4]). The sequence $s_{n}(x)$ in equation (3.12) is the Sheffer sequence for the pair $(g(t), f(t))$ if and only if they admit the exponential generating function of the form

$$
\frac{1}{g(\bar{f}(t))} e^{x(\bar{f}(t))}=\sum_{n=0}^{\infty} s_{n}(x) \frac{t^{n}}{n !},
$$

where $\bar{f}(t)$ is the compositional inverse of $f(t)$.

Now, consider the class $\mathscr{P}$ of polynomials $P_{n}$. Let $G$ denote the generating function, i.e.,

$$
G(t, x):=\sum_{n \geq 0} P_{n}(x) \frac{t^{n}}{n !} .
$$

Theorem 3.3. For the random variable $F_{\infty}=N_{1} \times N_{2}$ consider the associated family of polynomials $\mathscr{P}$ given by (1.4). Then, the family $\mathscr{P}$ is Sheffer for the pair

$$
(f(t), g(t))=\left(\operatorname{coth}^{-1}\left(\frac{1}{t}\right), \frac{1}{\sqrt{1-t^{2}}}\right) .
$$

Proof. Note that the generating function $G$ satisfies in the following second order PDE:

$$
\frac{d}{d x}\left(x \frac{d}{d x} G(t, x)\right)=x G(t, x)-\frac{d}{d t} G(t, x),
$$

i.e.,

$$
G_{t}=x G-G_{x}-x G_{x x} .
$$

Hence, the PDE (3.14) is just the associated PDE, scaled in space, in the Feynman-Kac formula for the squared Bessel process with index $\delta=2$, and therefore [10, Theorem 5.4.2] immediately implies that

$$
G(t, x)=\frac{e^{x \cdot \operatorname{coth}(t)}}{\cosh (t)} .
$$

Therefore $f(t)=\operatorname{coth}^{-1}(1 / t)$ in the representation (3.13), and moreover, $g(t)=\frac{1}{\sqrt{1-t^{2}}}$ is a direct consequence of the hyper-trigonometric identity $\sinh \left(\cosh ^{-1}(t)\right)=\sqrt{t^{2}-1}$.

A polynomial set $\mathscr{P}=\left\{P_{n}\right\}_{n \geq 0}$ (i.e., $\operatorname{deg}\left(P_{n}\right)=n$ for all $n \geq 0$ ) is said to be quasimonomial if there are two operators $\mathfrak{R}$, and $\mathfrak{L}$ independent of $n$ such that

$$
\mathfrak{R}\left(P_{n}\right)(x)=P_{n+1}(x) \text { and } \mathfrak{L}\left(P_{n}\right)(x)=P_{n-1}(x) .
$$

In other words, operators $\mathfrak{R}$ and $\mathfrak{L}$ play similar roles to the multiplicative and derivative operators, respectively, on monomials. We refer to the $\mathfrak{L}$ and $\mathfrak{R}$ operators as the descending (or lowering) and ascending (or raising) operators associated with the polynomial set $\mathscr{P}$. A fundamental result (see [12, Theorem 2.1]) states that every polynomial set is quasi-monomial in the above sense. The next corollary aims to take advantage of being a 
Sheffer polynomial set $\mathscr{P}$ to provide an explicit form of the associated lowering operator $\mathfrak{L}$.

Corollary 3.1. For the Sheffer family $\mathscr{P}$ of polynomials $P_{n}$ associated to random variable $F_{\infty}=N_{1} \times N_{2}$, the lowering operator, i.e., $\mathfrak{L} P_{n}=n P_{n-1}$ for $n \geq 1$ is given by

$$
\mathfrak{L}=f(D), \quad \text { and } \quad f(t)=\operatorname{coth}^{-1}\left(\frac{1}{t}\right)=\sum_{k \geq 0} \frac{t^{2 k+1}}{2 k+1},
$$

where $D$ stands for derivative operator.

Proof. This is an application of [27, Theorem 2.3.7].

The polynomial set $\mathscr{P}$ appearing in Corollary 3.1 is called the $\mathfrak{L}$-Appell polynomial set since

$$
\mathfrak{L} P_{n}=n P_{n-1} \quad \forall n \geq 1 .
$$

This notion generalizes the classical concept of the Appell polynomial set meaning that

$$
\frac{d}{d x} P_{n}(x)=n P_{n-1}(x)
$$

for every $n \geq 1$. Among the classical Appell polynomial set, we recall the monomials set $\left\{x^{n}\right\}_{n \geq 0}$, Hermite polynomials, the Bernoulli polynomials, and the Euler polynomials. For the application of Appell polynomials in non-central probabilistic limit theorems, we refer the reader to [4.

The next corollary provides more information on the constant coefficients of the even degree polynomials $P_{n} \in \mathscr{P}$.

Corollary 3.2. Let $\mathscr{P}=\left\{P_{n}: n \geq 1\right\}$ be the associated Sheffer family of the random variable $F_{\infty}=N_{1} \times N_{2}$. Assume that $E_{n}, n \geq 1$ stands for Euler numbers (see [15, Section 1.14, p. 48]). Then

$$
\mathfrak{a}(2 n, 0)=E_{2 n}, \quad n \geq 1 .
$$

Hence, the following representation of even Euler numbers is in order, for $n \in \mathbb{N}$ even number,

$$
E_{n}=(-1)^{n / 2} \sum_{i_{1}=1}^{1} i_{1}^{2} \sum_{i_{2}=1}^{i_{1}+1} i_{2}^{2} \sum_{i_{3}=1}^{i_{2}+1} i_{3}^{2} \cdots \sum_{i_{n / 2}=1}^{i_{n / 2-1}+1} i_{n / 2}^{2} .
$$

Proof. According to [27, Theorem 2.3.5],

$$
P_{n}(x)=\sum_{k=0}^{n} \frac{1}{k !}\left\langle g(\bar{f}(t))^{-1} f(t)^{k} \mid x^{n}\right\rangle x^{k} .
$$

Hence, the result follows at once by taking into account Theorem 3.3, and Taylor series expansion of Euler numbers

$$
\frac{1}{\cosh (t)}=\frac{2 e^{t}}{e^{2 t}+1}=\sum_{n \geq 0} E_{n} \frac{t^{n}}{n !} .
$$

3.3. Orthogonal Sheffer polynomial sequences. There are several characterizations of the orthogonal Sheffer polynomial sequences. Here we mentioned the one in terms of their generating function originally due to Sheffer (1939) 31. Consider a Sheffer polynomial sequence $\left\{s_{n}(x)\right\}_{n \geq 0}$ associated to the pair $(g(t), f(t))$, see Theorem 3.2 , with the generating function

$$
G(t, x)=\frac{1}{g(\bar{f}(t))} e^{x(\bar{f}(t))}=\sum_{n=0}^{\infty} s_{n}(x) \frac{t^{n}}{n !} .
$$


Theorem 3.4. A Sheffer polynomial sequence $\left\{s_{n}(x)\right\}_{n \geq 0}$ is orthogonal if and only if its generating function $G(t, x)$ is one of the following forms:

$$
\begin{aligned}
G(t, x) & =\mu(1-b t)^{c} \exp \left\{\frac{d+a t x}{1-b t}\right\}, & & a b c \mu \neq 0, \\
G(t, x) & =\mu \exp \left\{t(b+a x)+c t^{2}\right\}, & & a c \mu \neq 0, \\
G(t, x) & =\mu e^{c t}(1-b t)^{d+a x}, & & a b c \mu \neq 0, \\
g(t, x) & =\mu\left(1-\frac{t}{c}\right)^{d_{1}+x / a}\left(1-\frac{t}{b}\right)^{d_{2}-x / a}, & & a b c \mu \neq 0, b \neq c .
\end{aligned}
$$

Among the well-known Sheffer orthogonal polynomial sequences are Laguerre, Hermite, Charlier, Meixner, Meixner-Pollaczek, and Krawtchouk polynomials. See the excellent textbooks [14,20] for definitions and more information. Theorem 3.4 can be directly performed to give an alternative proof of Proposition 3.2. The reader is also referred to references [13, 33 for related results on the associated orthogonal polynomials with respect to the probability measure $F_{\infty}=N_{1} \times N_{2}$ on the real line.

Corollary 3.3. Let $F_{\infty}=N_{1} \times N_{2}$, where $N_{1}, N_{2} \sim \mathscr{N}(0,1)$ are independent, and the associated polynomials set $\mathscr{P}$ is given by (1.4). Then polynomial family $\mathscr{P}$ is Sheffer but not orthogonal.

Remark 3.2 ([31]). A necessary and sufficient condition for $\left\{s_{n}(x)\right\}_{n \geq 0}$ to be an orthogonal Sheffer family is that the monic recursion coefficients $a_{n}$ and $b_{n}$ in the three-term recurrence relation $s_{n+1}(x)=\left(x-a_{n}\right) s_{n}(x)-b_{n} s_{n-1}(x)$ have the form

$$
a_{n+1}=c_{1}+c_{2} n \quad \text { and } \quad b_{n+1}=c_{3} n+c_{4} n^{2}, \quad c_{1}, \ldots, c_{4} \in \mathbb{R}
$$

with $b_{n+1}>0$, in other words $a_{n}$ is at most linear in $n$ and that $b_{n}$ is at most quadratic in $n$.

\section{Connection with weak Convergence on the Second Wiener chaos}

4.1. Normal approximation with higher even moments. The aim of this section is to build a bridge between the Sheffer family of polynomials $\mathscr{P}$ given by (1.4) associated to target random variable $F_{\infty}=N_{1} \times N_{2}$, and the non-central limit theorems on the second Wiener chaos. We start with a striking result appearing in 2005 known nowadays as the fourth moment theorem due to Nualart and Peccati [25] stating that for a normalized sequence $\left\{F_{n}\right\}_{n \geq 1}$, meaning that $\mathrm{E}\left[F_{n}^{2}\right]=1$ for all $n \geq 1$, in a fixed Wiener chaos of order $p \geq 2$, the weak convergence $F_{n}$ towards $\mathscr{N}(0,1)$ is equivalent with convergence of the fourth moments $\mathrm{E}\left[F_{n}^{4}\right] \rightarrow 3\left(=\mathrm{E}\left[\mathscr{N}(0,1)^{4}\right]\right)$. In the case of normal approximation on the Wiener chaos, the authors of [6] introduced a novel family of special polynomials that characterizes the weak convergence of the sequence $\left\{F_{n}\right\}_{n \geq 1}$ towards standard normal distribution. Assume that $\left\{F_{n}\right\}_{n \geq 1}$ is a sequence of random elements in the fixed Wiener chaos of order $p \geq 2$ such that $\mathrm{E}\left[F_{n}^{2}\right]=1$ for all $n \geq 1$. Following [6] we consider the family $\mathscr{Q}_{\geq 0}$ of polynomials defined as follows: for every $k \geq 2$, define the monic polynomial $W_{k}$ as

$$
W_{k}(x)=(2 k-1)\left(x \int_{0}^{x} H_{k}(t) H_{k-2}(t) d t-H_{k}(x) H_{k-2}(x)\right),
$$

where $H_{k}$ is the $k$ th Hermite polynomial, and set

$$
\mathscr{Q}_{\geq 0}:=\left\{P \mid P(x)=\sum_{k=2}^{m} \alpha_{k} W_{k}(x) ; m \geq 2, \alpha_{k} \geq 0\right\} .
$$


Then, one of the main findings of [6] states that the polynomial family $\mathscr{Q}_{\geq 0}$ characterizes the normal approximation on the Wiener chaos in the sense that the following statements are equivalent:

(I): $F_{n} \stackrel{\text { law }}{\longrightarrow} \mathscr{N}(0,1)$.

(II): $(0 \leq) \mathrm{E}\left[P\left(F_{n}\right)\right] \rightarrow \mathrm{E}[P(\mathscr{N}(0,1))](=0)$ for some $P \in \mathscr{Q}_{\geq 0}$.

One of the significant consequences of the aforementioned equivalence is the following generalization of the Nualart-Peccati fourth moment criterion. The symbol $d_{T V}$ stands for the total variation distance.

Theorem 4.1 (Even moment theorem [6]). Let $\left\{F_{n}\right\}_{n \geq 1}$ be a sequence of random elements in the fixed Wiener chaos of order $p \geq 2$, and that $\mathrm{E}\left[F_{n}^{2}\right]=1$ for all $n \geq 1$. Let $N \sim \mathscr{N}(0,1)$. Then the following asymptotic statements are equivalent:

(I): $F_{n} \stackrel{\text { law }}{\longrightarrow} N$.

(II): $m_{2 k}\left(F_{n}\right):=\mathrm{E}\left[F_{n}^{2 k}\right] \rightarrow m_{2 k}(N)=(2 k-1) ! !$.

Furthermore, for some constant $C$, independent of $n$, the following estimate in the total variation probability metric takes place:

$$
d_{T V}\left(F_{n}, \mathscr{N}(0,1)\right) \leq C \sqrt{\mathrm{E}\left[F_{n}^{2 k}\right]-(2 k-1) ! !} .
$$

4.2. Convergence towards $N_{1} \times N_{2}$ : cumulants criterion. In what follows, $D$ and $L\left(L^{-1}\right)$ stand for the Malliavin derivative operator, the infinitesimal Ornstein-Uhlenbeck generator (the pseudo-inverse of operator $L$ ), respectively. Next, we define the iterated Gamma operators (see the excellent monograph 23 for a complete overview on the topic as well as the non-explained notation) as follows: for a "smooth" random variable $F$ in the sense of Malliavin calculus, define $\Gamma_{0}(F)=F$, and $\Gamma_{r}(F)=\left\langle D F,-D L^{-1} \Gamma_{r-1}(F)\right\rangle_{\mathfrak{H}}$ for $r \geq 1$, where $\mathfrak{H}$ is the underlying separable Hilbert space. Also, the useful fact $\kappa_{r}(F)=(r-1) ! \mathrm{E}\left[\Gamma_{r-1}(F)\right]$ is well known, see [23, Theorem 8.4.3], where $\kappa_{r}(F)$ stands for the $r$ th cumulant of the random variable $F$.

We continue with the following non-central convergence towards the target distribution $F_{\infty}=N_{1} \times N_{2}$ in terms of the convergences of finitely many cumulants/moments. In the framework of the second Wiener chaos, it has been first proven in [24] using the method of complex analysis. For a rather general setup using the iterated Gamma operators and the Malliavin integration by parts formulae, see [7]. Also, for quantitative Berry-Esseen estimates see the recent works [3, 19, and [5, 16] for the free counterpart statements.

Theorem 4.2. Let $\left\{F_{n}\right\}_{n \geq 1}$ be a centered sequence of random elements in a finite direct sum of Wiener chaos such that $\mathrm{E}\left[F_{n}^{2}\right]=1$ for all $n \geq 1$. Then, the following statements are equivalent:

(I): sequence $F_{n}$ converges in distribution towards $F_{\infty} \sim N_{1} \times N_{2}$;

(II): as $n \rightarrow \infty$ the following asymptotic relations hold:

(1) $\kappa_{3}\left(F_{n}\right) \rightarrow 0$

(2) $\Delta\left(F_{n}\right):=\operatorname{Var}\left(\Gamma_{2}\left(F_{n}\right)-F_{n}\right) \rightarrow 0$.

Whenever the sequence $\left\{F_{n}\right\}_{n \geq 1}$ belongs to the second Wiener chaos, the quantity $\Delta\left(F_{n}\right)$ appearing in item $(2)$ can be replaced with

$$
\Delta^{\prime}\left(F_{n}\right)=\frac{\kappa_{6}\left(F_{n}\right)}{5 !}-2 \frac{\kappa_{4}\left(F_{n}\right)}{3 !}+\kappa_{2}\left(F_{n}\right) .
$$

The following proposition aims to provide a direct link between the Sheffer polynomial class $\mathscr{P}$ and Theorem 4.2 . The Wasserstein-2 distance between two probability distributions $Q_{1}, Q_{2}$ on $(\mathbb{R}, \mathcal{B}(\mathbb{R}))$ is given by

$$
d_{W_{2}}\left(Q_{1}, Q_{2}\right):=\inf _{\left(X_{1}, X_{2}\right)}\left\{\mathrm{E}\left(\left(X_{1}-X_{2}\right)^{2}\right)^{1 / 2}\right\}
$$


where the supremum is taken over the random pairs $\left(X_{1}, X_{2}\right)$ defined on the same classical probability spaces $(\Omega, \mathcal{F}, \mathrm{P})$ with marginal distributions $Q_{1}$ and $Q_{2}$.

Proposition 4.1. Let $\left\{F_{n}\right\}_{n \geq 1}$ be a sequence in the second Wiener chaos such that $\mathrm{E}\left[F_{n}^{2}\right]=1$ for all $n \geq 1$. Consider polynomial $P_{6}(x)=x^{6}-55 x^{4}+331 x^{2}-61 \in \mathscr{P}$. Then, as $n$ tends to infinity, the following statements are equivalent:

(I): sequence $F_{n}$ converges in distribution towards $F_{\infty} \sim N_{1} \times N_{2}$.

(II): $\mathrm{E}\left[F_{n}^{4}\right] \rightarrow 9$, and $\mathrm{E}\left[F_{n}^{6}\right] \rightarrow 225$.

(III): $(0 \leq) \mathrm{E}\left[P_{6}\left(F_{n}\right)\right] \rightarrow \mathrm{E}\left[P_{6}\left(F_{\infty}\right)\right](=0)$.

In other words, polynomial $P_{6}$ captures at the same time the two necessary and sufficient conditions for convergence towards $F_{\infty}$ appearing in Theorem 4.2. Furthermore, the following quantitive estimate in Wasserstein-2 distance holds: for $n \geq 1$,

$$
d_{W_{2}}\left(F_{n}, F_{\infty}\right) \leq_{C} \sqrt{P_{6}\left(F_{n}\right)} \leq_{C} \sqrt{\left(\mathrm{E}\left[F_{n}^{6}\right]-225\right)-55\left(\mathrm{E}\left[F_{n}^{4}\right]-9\right)} .
$$

Proof. Implication $(\mathbf{I}) \Rightarrow(\mathbf{I I})$ is just an application of the continuous mapping theorem, and the hypercontractivity property of the Wiener chaoses (see [23, Theorem 2.7.2, p. 36]). $(\mathbf{I I}) \Leftrightarrow$ (III) Using the relation between moments and cumulants of random variables (see [26. p. 259]) and straightforward computation, and taking into account that $\mathrm{E}\left[P_{6}\left(F_{\infty}\right)\right]=$ 0 , it yields that

$$
\begin{aligned}
\mathrm{E}\left[P_{6}\left(F_{n}\right)\right] & =\left(\mathrm{E}\left[F_{n}^{6}\right]-225\right)-55\left(\mathrm{E}\left[F_{n}^{4}\right]-9\right) \\
& =5 ! \Delta^{\prime}\left(F_{n}\right)+10 \kappa_{3}^{2}\left(F_{n}\right) \geq 0
\end{aligned}
$$

under the lights of item 2 of Theorem 4.2 , and the fact that $F_{n}$ is in the second Wiener chaos. Finally implication $(\mathbf{I I}) \Rightarrow(\mathbf{I})$ together with the estimate (4.4) is borrowed from [5. Proposition 5.1].

Remark 4.1. The following remarks are of independent interest. Let $F$ be a random element in the second Wiener chaos with the second moment $\mathrm{E}\left[F^{2}\right]=1$.

(a) The crucial relations

$$
(0 \leq) \mathrm{E}\left[P_{6}(F)\right]=5 ! \Delta^{\prime}(F)+10 \kappa_{3}^{2}(F)=5 ! \operatorname{Var}\left(\Gamma_{2}(F)-F\right)+10 \kappa_{3}^{2}(F)
$$

appearing in Proposition 4.1 can be directly deduced using the Malliavin integration by part formulae instead of the relation between moments and cumulants. In fact, using the very definition of polynomials in the family $\mathscr{P}$ through the rising operator $\mathfrak{R}$, and also applying twice the Malliavin integration by parts formula [23, Theorem 2.9.1], we can write (to follow incoming computation, one has to note that $\mathrm{E}[F]=0$, and $\mathrm{E}\left[\Gamma_{1}(F)\right]=$ $\left.\mathrm{E}\left[F^{2}\right]=1\right)$

$$
\begin{aligned}
\mathrm{E}\left[P_{6}(F)\right] & =\mathrm{E}\left[\Re P_{5}(F)\right]=\mathrm{E}\left[F P_{5}(F)-P_{5}^{\prime}(F)-F P_{5}^{\prime \prime}(F)\right] \\
& =\mathrm{E}\left[P_{5}^{\prime \prime}(F)\left(\Gamma_{2}(F)-\mathrm{E}\left[\Gamma_{2}(F)\right]-F\right)\right]+\mathrm{E}\left[\Gamma_{2}(F)\right] \mathrm{E}\left[P_{5}^{\prime \prime}(F)\right] \\
& =\mathrm{E}\left[P_{5}^{\prime \prime}(F)\left(\Gamma_{2}(F)-\mathrm{E}\left[\Gamma_{2}(F)\right]-F\right)\right]+10 \kappa_{3}^{2}(F) .
\end{aligned}
$$

As a direct consequence, in order to have $\operatorname{Var}\left(\Gamma_{2}(F)-F\right)$ in the very last right hand side of relation (4.6), there must be one more copy of the random variable $\Gamma_{2}(F)-\mathrm{E}\left[\Gamma_{2}(F)\right]-F$ inside the quantity $P_{5}^{\prime \prime}(F)$. Now, note that for $F=I_{2}(f)$ being in the second Wiener chaos,

$$
\Gamma_{r}(F)-\mathrm{E}\left[\Gamma_{r}(F)\right]=I_{2}\left(2^{r} f \otimes_{1}^{(r+1)} f\right), \quad r \geq 1,
$$

which implies that random variable $\Gamma_{2}(F)-\mathrm{E}\left[\Gamma_{2}(F)\right]-F$ belongs to the second Wiener chaos. Now, taking into account orthogonality of the Wiener chaos, in order to compute $\mathrm{E}\left[P_{5}^{\prime \prime}(F)\left(\Gamma_{2}(F)-\mathrm{E}\left[\Gamma_{2}(F)\right]-F\right)\right]$, one needs only to understand the projection of random variable $P_{5}^{\prime \prime}(F)$ on the second Wiener chaos. Since, $F$ is a multiple integral, 
and $P_{5}^{\prime \prime}$ is a polynomial, so random variable $P_{5}^{\prime \prime}(F)$ is smooth in the sense of Malliavin differentiability. Hence, one can use Stroock's formula [23, Corollary 2.7.8] to compute the second projection. We have

$$
P_{5}(x)=x^{5}-30 x^{3}+61 x \quad \Rightarrow \quad P_{5}^{\prime \prime}(x)=20\left(x^{3}-9 x\right) .
$$

Hence, $P_{5}^{\prime \prime}(F)=\sum_{p=0}^{3} I_{p}\left(g_{p}\right)$, where

$$
g_{2}\left(t_{1}, t_{2}\right)=\frac{1}{2 !} \mathrm{E}\left[6 F\left(D_{t_{1}} F\right)\left(D_{t_{2}} F\right)+\left(3 F^{2}-9\right) D_{t_{1}, t_{2}}^{2} F\right] .
$$

For example,

$$
\begin{aligned}
\mathrm{E}\left[6 F\left(D_{t_{1}} F\right)\left(D_{t_{2}} F\right)\right] & =6 \times 2 \times 2 \mathrm{E}\left[I_{2}(f) \times I_{1}\left(f\left(t_{1}, \cdot\right)\right) \times I_{1}\left(f\left(t_{2}, \cdot\right)\right)\right] \\
& =24 \mathrm{E}\left[I_{2}(f) \times I_{2}\left(f\left(t_{1}, \cdot\right) \otimes f\left(t_{2}, \cdot\right)\right)\right] \\
& =24 \times 2\left\langle f, f\left(t_{1}, \cdot\right) \otimes f\left(t_{2}, \cdot\right)\right\rangle \\
& =6 \times 2^{3}\left(f \otimes_{1}^{(3)} f\right)\left(t_{1}, t_{2}\right) .
\end{aligned}
$$

Similar computations can be done for the other term. All together they imply that

$$
g_{2}\left(t_{1}, t_{2}\right)=5 !\left(2^{2}\left(f \otimes_{1}^{(3)} f\right)\left(t_{1}, t_{2}\right)-f\left(t_{1}, t_{2}\right)\right) .
$$

The latter is exactly the kernel of random element $\Gamma_{2}(F)-\mathrm{E}\left[\Gamma_{2}(F)\right]-F$.

(b) Assume that $F_{\infty} \sim N_{1} \times N_{2}$. Then

$$
x^{8}-11025=x^{8}-\mathrm{E}\left[F_{\infty}^{8}\right]=P_{8}(x)+140 P_{6}(x)+4214 P_{4}(x)+24940 P_{2}(x) .
$$

This in turn implies that

$$
\left\{m_{8}(F)-m_{8}\left(F_{\infty}\right)\right\}-4214\left\{m_{4}(F)-m_{4}\left(F_{\infty}\right)\right\}=\mathrm{E}\left[P_{8}(F)\right]+140 \mathrm{E}\left[P_{6}(F)\right] .
$$

However, in general, for a random element $F$ in the second Wiener chaos with $\mathrm{E}\left[F^{2}\right]=1$, unlike the quantity $\mathrm{E}\left[P_{6}(F)\right]$, the expectation $\mathrm{E}\left[P_{8}(F)\right]$ can take negative values, too. For example, assume that $G_{\infty}$ is an independent copy of $F_{\infty}$. Consider, for every $t \in[0,1]$, the random element

$$
F_{t}:=\sqrt{t} F_{\infty}+\sqrt{1-t} G_{\infty} .
$$

Note that $F_{t}$ belongs to the second Wiener chaos, and that law $\left(F_{t}\right) \neq \operatorname{law}\left(F_{\infty}\right)$ for every $t \in(0,1)$. Define the auxiliary function $Q_{4}(t):=\mathrm{E}\left[P_{8}\left(F_{t}\right)\right]$. Using MATLAB, we obtain that

$$
Q_{4}(t)=15120 t^{4}-30240 t^{3}+19152 t^{2}-4032 t .
$$

The graph of the polynomial $Q_{4}$ is shown in Figure 1. We note that $Q_{4}(t) \leq 0$ for every $t \in[0,1]$, and furthermore $Q_{4}(0)=\mathrm{E}\left[P_{8}\left(G_{\infty}\right)\right]=Q_{4}(1)=\mathrm{E}\left[P_{8}\left(F_{\infty}\right)\right]=0$. As a conclusion, this line of argument cannot be useful to justify the fact that convergences of the fourth and the eighth moments are enough to declare convergence in distribution. See [5], and also the forthcoming remark.

Definition 4.1. Let $F_{\infty} \sim N_{1} \times N_{2}$. We say that a polynomial $P$ (of degree $\geq 3$ ) characterizes the law of $F_{\infty}$ whenever $\mathrm{E}[P(F)]=0$ for some random element $F$ inside the second Wiener chaos so that $\mathrm{E}\left[F^{2}\right]=1$; then $F \sim F_{\infty}$. Also, we say that polynomial $P$ sequentially characterizes the law of $F_{\infty}$ whenever $\mathrm{E}\left[P\left(F_{n}\right)\right] \rightarrow 0$ for some normalized random sequence $\left\{F_{n}\right\}_{n \geq 1}$ inside the second Wiener chaos; then $F_{n} \rightarrow F_{\infty}$ in distribution. 


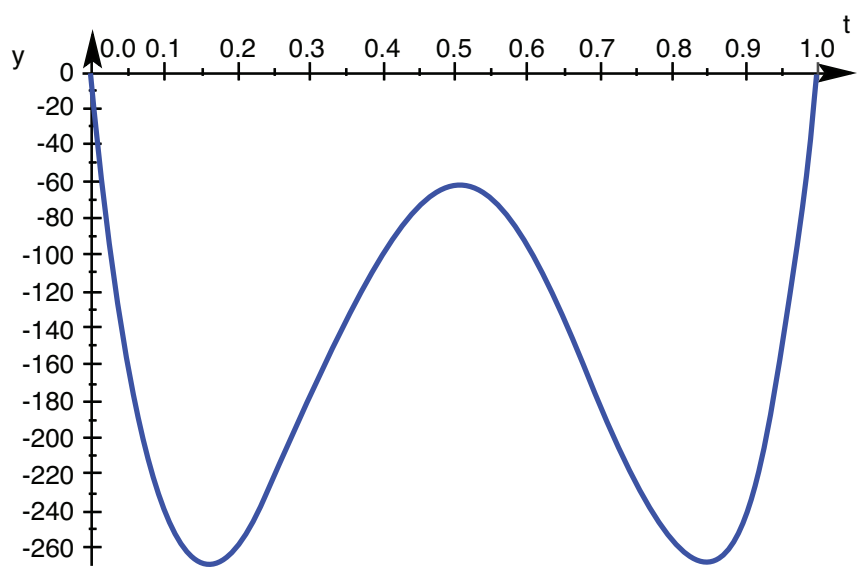

Figure 1. $Q_{4}(t)=15120 t^{4}-30240 t^{3}+19152 t^{2}-4032 t$

Remark 4.2. The aim of the remark is to clarify the role of other polynomials $P_{n} \in \mathscr{P}$ in the characterization of the normal product distribution in the sense of Definition 4.1. We have already shown that when $F$ is a normalized element in the second Wiener with $\mathrm{E}\left[P_{6}(F)\right]=0$, then $F$ is necessarily distributed as the normal product distribution. For the polynomial $P_{8}$, we consider the random element $F$ in the second Wiener chaos of the form

$$
F=-\frac{1}{\sqrt{3}}\left(N_{-1}^{2}-1\right)+\frac{1}{\sqrt{12}}\left(N_{1}^{2}-1\right)+\frac{1}{\sqrt{12}}\left(N_{2}^{2}-1\right)=\frac{N_{1}^{2}+N_{2}^{2}-2 N_{-1}^{2}}{\sqrt{12}} .
$$

We found the element $F$ by using a random search algorithm. Note that $\mathrm{E}\left[F^{2}\right]=1$, and some straightforward computations yield that $\mathrm{E}\left[P_{4}(F)\right]=\mathrm{E}\left[P_{8}(F)\right]=0$, and furthermore $\operatorname{law}(F) \neq \operatorname{law}\left(F_{\infty}\right)$ where $F_{\infty} \sim N_{1} \times N_{2}$. For the random variable $F$ in (4.7) we have that

$$
F \stackrel{\text { law }}{=} \frac{1}{\sqrt{3}}\left(G_{1} G_{2}+G_{3} G_{4}\right)
$$

where $\left(G_{1}, G_{2}, G_{3}, G_{4}\right)$ is a Gaussian vector with zero mean and covariance matrix

$$
\left(C_{i j}\right)_{1 \leq i, j \leq 4}=\left(\begin{array}{cccc}
1 & 0 & 1 & -1 \\
0 & 1 & -1 & 1 \\
1 & -1 & 1 & 0 \\
-1 & 1 & 0 & 1
\end{array}\right)
$$

with the Hankel matrix form $\mathrm{E}\left(G_{i} G_{j}\right)=C(i+j)$ with $C(2)=C(8)=1, C(3)=C(7)=$ $0, C(4)=C(6)=1, C(5)=-1$, is the normalized sum of two dependent copies of $N_{1} \times N_{2}$ random variables. One possibility for finding one root $\mathrm{E}\left[P_{2 n}(F)\right]=0$ other than normal product distribution for higher values of $n$ is to consider polynomials (see also item (b) of Remark 4.1)

$$
Q_{n}(t):=\mathrm{E}\left[P_{2 n}\left(F_{t}\right)\right]=\mathrm{E}\left[P_{2 n}\left(\sqrt{t} F_{\infty}+\sqrt{1-t} G_{\infty}\right)\right], \quad t \in[0,1],
$$

where $G_{\infty}$ is an independent copy of $F_{\infty}$. It is easy to see that $Q_{n}$ is a polynomial in $t$ so that $\operatorname{deg}\left(Q_{n}\right)=n$ when $n$ is even, and $\operatorname{deg}\left(Q_{n}\right)=n-1$ when $n$ is odd, and hence $\operatorname{deg}\left(Q_{n}\right)$ is always even. See Figure 2 for the graphs of polynomials $Q_{n}$, for $n=5,6,7$ on the interval $[0,1]$. It can be seen that polynomials $Q_{5}, Q_{6}$, and $Q_{7}$ have at least one real root in the open interval $(0,1)$. Moreover, for every $n \geq 5$ one can show that polynomial 
$Q_{n}$ is of the form

$$
Q_{n}(t)= \begin{cases}\sum_{k=1}^{n}(-1)^{k} \mathfrak{b}(n, k) t^{k}, & n \text { even, } \\ \sum_{k=1}^{n-1}(-1)^{k-1} \mathfrak{b}(n, k) t^{k}, & n \text { odd }\end{cases}
$$

where coefficients $\mathfrak{b}(n, k)$ are all positive (non-zero) real numbers for every $1 \leq k \leq n$ (or $n-1$ ) depending on whether $n$ is even or odd. Hence, as a direct consequence the number of sign changes in $Q_{n}(x+0)=Q_{n}(x)=\operatorname{deg}\left(Q_{n}\right)-1$ which is always an odd number. Furthermore $Q_{n}(0)=Q_{n}(1)=0$. This directs one to the possible use of the Budan-Fourier theorem on the positive real roots of polynomials, and we leave it for further investigation. Finally, note that since the distribution $F_{\infty}$ is symmetric, and polynomials $P_{k} \in \mathscr{P}$ for $k \geq 3$ being odd contain only odd powers of $x$, so the odd degree polynomials $P_{n}$ cannot be used in characterization of $F_{\infty}$ in the above sense.
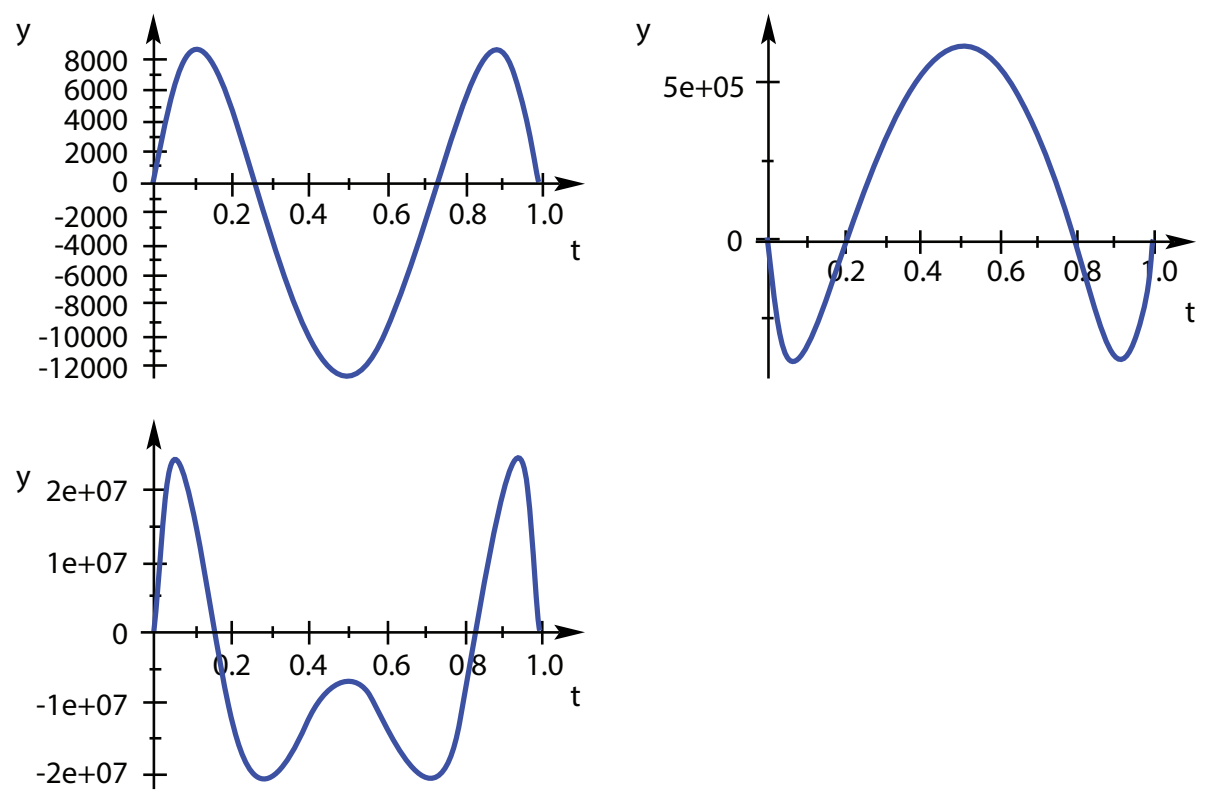

FIGURE 2. Graphs of polynomials $Q_{5}, Q_{6}, Q_{7}$ 


\section{Appendix A}

$$
\begin{aligned}
& P_{0}(x)=1, \\
& P_{1}(x)=x, \\
& P_{2}(x)=x^{2}-1 \text {, } \\
& P_{3}(x)=x^{3}-5 x, \\
& P_{4}(x)=x^{4}-14 x^{2}+5 \text {, } \\
& P_{5}(x)=x^{5}-30 x^{3}+61 x, \\
& P_{6}(x)=x^{6}-55 x^{4}+331 x^{2}-61 \text {, } \\
& P_{7}(x)=x^{7}-91 x^{5}+1211 x^{3}-1385 x, \\
& P_{8}(x)=x^{8}-140 x^{6}+3486 x^{4}-12284 x^{2}+1385 \text {, } \\
& P_{9}(x)=x^{9}-204 x^{7}+8526 x^{5}-68060 x^{3}+50521 x \text {, } \\
& P_{10}(x)=x^{10}-285 x^{8}+18522 x^{6}-281210 x^{4}+663061 x^{2}-50521 \text {, } \\
& P_{11}(x)=x^{11}-385 x^{9}+36762 x^{7}-948002 x^{5}+5162421 x^{3}-2702765 x, \\
& P_{12}(x)=x^{12}-506 x^{10}+67947 x^{8}-2749340 x^{6}+28862471 x^{4}-49164554 x^{2}+2702765 \text {, } \\
& P_{13}(x)=x^{13}-650 x^{11}+118547 x^{9}-7097948 x^{7}+127838711 x^{5}-510964090 x^{3} \\
& +199360981 x \\
& P_{14}(x)=x^{14}-819 x^{12}+197197 x^{10}-16700255 x^{8}+475638163 x^{6}-3706931865 x^{4} \\
& +4798037791 x^{2}-199360981 \text {, } \\
& P_{15}(x)=x^{15}-1015 x^{13}+315133 x^{11}-36419955 x^{9}+1544454483 x^{7}-20829905733 x^{5} \\
& +64108947633 x^{3}-19391512144 x \text {. }
\end{aligned}
$$

\section{Appendix B}

Lemma 6.1. Consider a random variable $X \in L^{1}(P)$ and a random vector $Y \in \mathbb{R}^{d}$ with continuous density $p_{Y}(u)$. Then

$$
\mathrm{E}(X \mid Y=u)=\frac{\mathrm{E}\left(X \delta_{u}(Y)\right)}{\mathrm{E}\left(\delta_{u}(Y)\right)} \in L^{1}\left(\mathbb{R}^{d}, P_{Y}\right),
$$

where $\delta_{u}(y)=\delta_{0}(y-u), \delta_{0}$ is the Dirac delta function, and $\mathrm{E}\left(\delta_{u}(Y)\right)=p_{Y}(u)$ is the density of $Y$.

Proof. Note first that by definition of the Dirac delta

$$
\mathrm{E}\left(\delta_{u}(Y)\right)=\int_{\mathbb{R}^{d}} \delta_{u}(y) p_{Y}(y) d y=p_{Y}(u)
$$

Also, for any bounded continuous test function $g: \mathbb{R}^{d} \rightarrow \mathbb{R}$

$$
\begin{aligned}
\mathrm{E}(X g(Y)) & =\int_{\Omega}\left(\int_{\mathbb{R}^{d}} \delta_{0}(Y(\omega)-u) g(u) d u\right) X(\omega) \mathrm{P}(d \omega) \\
& =\int_{\mathbb{R}^{d}} \frac{\mathrm{E}\left(X \delta_{0}(Y-u)\right)}{p_{Y}(u)} g(u) p_{Y}(u) d u
\end{aligned}
$$


Using the Fubini theorem with the generalized function $\delta_{0}$ is justified as follows: for a sequence of mollifiers with compact support $\eta_{n} \rightarrow \delta_{0}$ (in distribution), for example

$$
\eta_{n}(x)=n^{d} \prod_{i=1}^{d}\left(1-n\left|x_{i}\right|\right)^{+},
$$

we have

$$
\begin{gathered}
\int_{\mathbb{R}^{d}} \frac{\mathrm{E}\left(\eta_{n}(Y-u) X\right)}{p_{Y}(u)} g(u) p_{Y}(u) d u=\int_{\Omega}\left(\int_{\mathbb{R}^{d}} \eta_{n}(Y(\omega)-u) g(u) d u\right) X(\omega) \mathrm{P}(d \omega) \\
\longrightarrow \int_{\Omega}\left(\int_{\mathbb{R}^{d}} \delta_{0}(Y(\omega)-u) g(u) d u\right) X(\omega) \mathrm{P}(d \omega)
\end{gathered}
$$

since the measure $\mathrm{P}(d \omega) \eta_{n}(Y(\omega)-u) d u$ converges in distribution to $\mathrm{P}(d \omega) \delta_{Y(\omega)}(u) d u$. Note also that the sequence of functions

$$
\rho_{n}(u)=p_{Y}(u)^{-1} \mathrm{E}\left(\eta_{n}(Y(\omega)-u) X\right)
$$

is bounded in $L^{1}\left(\mathbb{R}^{d}, P_{Y}\right)$, and since the unit ball of $L^{1}\left(\mathbb{R}^{d}, P_{Y}\right)$ is weakly compact, the sequence $\rho_{n}(u)$ converges weakly in $L^{1}\left(\mathbb{R}^{d}, P_{Y}\right)$ towards (6.1) which satisfies (6.2). The results extend to all bounded measurable $g$ by the standard monotone class argument.

\section{ACKNOWLEDGMENT}

The authors would like to thank an anonymous referee for several constructive comments that significantly improved the presentation of our work.

\section{BIBLIOGRAPHY}

[1] M. Abramowitz and I. A. Stegun, eds., Modified Bessel Functions I and K, Handbook of Mathematical Functions with Formulas, Graphs, and Mathematical Tables, 9th ed., Dover, New York, 1972, pp. 374-377. MR1225604

[2] B. Arras, E. Azmoodeh, G. Poly, and Y. Swan, Stein characterizations for linear combinations of gamma random variables, 2017, https://arxiv.org/pdf/1709.01161.pdf.

[3] B. Arras, E. Azmoodeh, G. Poly, and Y. Swan, A bound on the 2-Wasserstein distance between linear combinations of independent random variables, Stochastic Process. Appl. 129 (2019), no. 7, 2341-2375. MR 3958435

[4] F. Avram and M. Taqqu, Noncentral limit theorems and Appell polynomials, Ann. Probab. 15 (1987), no. 2, 767-775. MR885142

[5] E. Azmoodeh and D. Gasbarra, New moments criteria for convergence towards normal product/tetilla laws, 2017, https://arxiv.org/abs/1708.07681.

[6] E. Azmoodeh, D. Malicet, G. Mijoule, and G. Poly, Generalization of the Nualart-Peccati criterion, Ann. Probab. 44 (2016), no. 2, 924-954. MR 3474463

[7] E. Azmoodeh, G. Peccati, and G. Poly, Convergence towards linear combinations of chi-squared random variables: a Malliavin-based approach, Séminaire de Probabilités XLVII (Special volume in memory of Marc Yor), 2014, 339-367. MR3444306

[8] S. Bai and M. Taqqu, Behavior of the generalized Rosenblatt process at extreme critical exponent values, Ann. Probab. 45 (2017), no. 2, 1278-1324. MR3630299

[9] V. Bally, Introduction to Malliavin Calculus, 2007, http://perso-math.univ-mlv.fr/users/bally . vlad/Osaka100407.pdf.

[10] J. Baldeaux and E. Platen, Functionals of Multidimensional Diffusions with Applications to Finance, Bocconi \& Springer Series, vol. 5, Springer \& Bocconi University Press, 2013. MR3113191

[11] Á. Baricz and S. Ponnusamy, On Turán type inequalities for modified Bessel functions, Proc. Amer. Math. Soc. 141 (2013), no. 2, 523-532. MR2996956

[12] Y. Ben Cheikh, Some results on quasi-monomiality, Appl. Math. Comput. 141 (2003), no. 1, 63-76. MR:1984228

[13] Y. Ben Cheikh and K. Douak, On two-orthogonal polynomials related to the Bateman's $J_{n}^{u, v}$ function, Methods Appl. Anal. 7 (2009), no. 4, 641-662. MR 1868550

[14] T. S. Chihara, An Introduction to Orthogonal Polynomials, Dover Books on Mathematics, 2011. MR0481884 
[15] L. Comtet, Advanced Combinatorics. The Art of Finite and Infinite Expansions, D. Reidel Publishing Co., 1974. MR0460128

[16] A. Deya and I. Nourdin, Convergence of Wigner integrals to the tetilla law, ALEA Lat. Am. J. Probab. Math. Stat. 9 (2012), 101-127. MR2893412

[17] R. E. Gaunt, On Stein's method for products of normal random variables and zero bias couplings, Bernoulli 23 (2017), no. 4B, 3311-3345. MR3654808

[18] R. E. Gaunt, Variance-Gamma approximation via Stein's method, Electron. J. Probab. 19 (2014), no. 38, 1-33. MR.3194737

[19] P. Eichelsbacher and C. Thäle, Malliavin-Stein method for Variance-gamma approximation on Wiener space, Electron. J. Probab. 20 (2015), no. 123, 1-28. MR3425543

[20] M. E. H. Ismail, Classical and Quantum Orthogonal Polynomials in One Variable, Encyclopedia of Mathematics and its Applications, vol. 98, Cambridge University Press, 2009. MR2542683

[21] S. Gradshetyn and I. M. Ryzhik, Table of Integrals, Series and Products, 7th ed., Academic Press, 2007. MR669666

[22] P. Malliavin, Integration and Probability, Graduate Texts in Mathematics, vol. 157, Springer-Verlag, New York, 1995. MR1335234

[23] I. Nourdin and G. Peccati, Normal Approximations Using Malliavin Calculus: from Stein's Method to Universality, Cambridge Tracts in Mathematics, vol. 192, Cambridge University Press, 2012. MR2962301

[24] I. Nourdin and G. Poly, Convergence in law in the second Wiener/Wigner chaos, Elect. Comm. in Probab. 17 (2012), no. 36. MR2970700

[25] D. Nualart and G. Peccati, Central limit theorems for sequences of multiple stochastic integrals, Ann. Probab. 330 (2005), no. 1, 177-193. MR2118863

[26] G. Peccati and M. S. Taqqu, Wiener Chaos: Moments, Cumulants and Diagrams, Bocconi \& Springer Series, vol. 1, Springer, Milan, 2011. MR2791919

[27] S. Roman, The Umbral Calculus, Pure and Applied Mathematics, vol. 111, Academic Press, New York, 1984. MR741185

[28] S. Roman, The theory of the umbral calculus, I. J. Math. Anal. 87 (1982), no. 1, 58-115. MR653607

[29] S. Roman and G. Rota, The Umbral Calculus, Advances Math. 27 (1978), 95-188. MR0485417

[30] R. J. Serfling, Approximation Theorems of Mathematical Statistics, John Wiley \& Sons, 1980. MR.595165

[31] I. M. Sheffer, Some properties of polynomial sets of type zero, Duke Math. J. 5 (1939), 590-622. MR 0000081

[32] M. D. Springer and W. E. Thompson, The distribution of products of Beta, Gamma and Gaussian random variables, SIAM J. Appl. Math. 18 (1970), 721-737. MR0264733

[33] W. Van Assche and S. B. Yakubovich, Multiple orthogonal polynomials associated with Macdonald functions, Integral Transforms Special Funct. 9 (2000), 229-244. MR1782974

Fakultät für Mathematik, Ruhr-Universität Bochum, N-Süd UG/7, 44780 Bochum, Germany Email address: ehsan.azmoodeh@rub.de

Department of Mathematics and Statistics, University of Helsinki, P.O. Box 68 (Gustaf HÄLlströmin KATU 2B) FI-00014 Helsinki, Finland

Email address: dario.gasbarra@helsinki.fi

Received 19/FEB/2018

Originally published in English 